This item was submitted to Loughborough's Research Repository by the author.

Items in Figshare are protected by copyright, with all rights reserved, unless otherwise indicated.

\title{
Regionalgeschichte und die Erforschung des Nationalsozialismus. Forschungsstand und Forschungsperspektiven
}

PLEASE CITE THE PUBLISHED VERSION

PUBLISHER

(C) Vandenhoeck \& Ruprecht

VERSION

AM (Accepted Manuscript)

LICENCE

CC BY-NC-ND 4.0

\section{REPOSITORY RECORD}

Szejnmann, Claus-Christian W.. 2019. "Regionalgeschichte Und Die Erforschung Des Nationalsozialismus. Forschungsstand Und Forschungsperspektiven”. figshare. https://hdl.handle.net/2134/14328. 
This item was submitted to Loughborough's Institutional Repository (https://dspace.lboro.ac.uk/) by the author and is made available under the following Creative Commons Licence conditions.

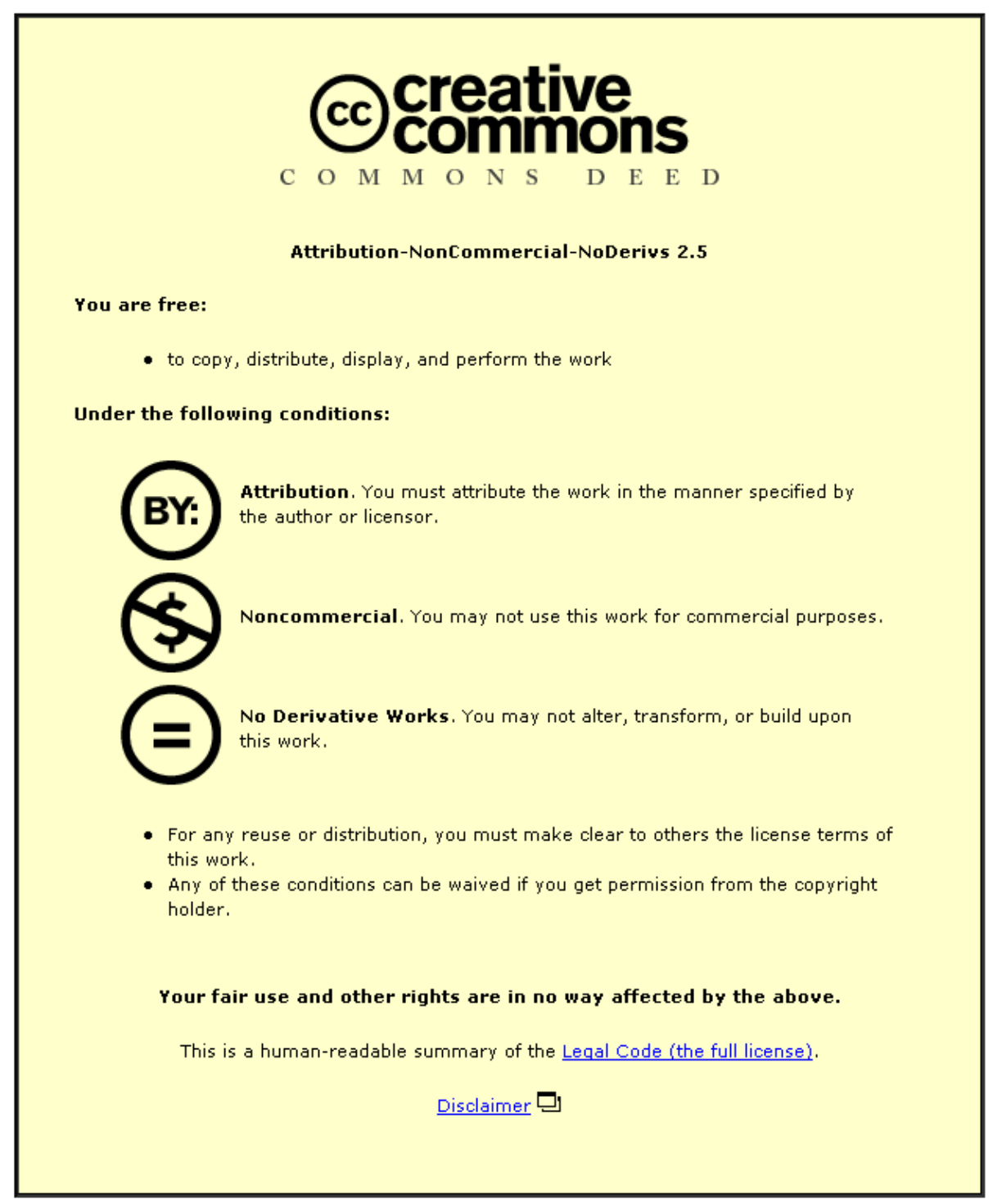

For the full text of this licence, please go to: http://creativecommons.org/licenses/by-nc-nd/2.5/ 


\section{Regionalgeschichte und die Erforschung des Nationalsozialismus - Forschungsstand und Forschungsperspektiven}

\section{Claus-Christian W. Szejnmann}

Fachleute klagen oft: „Die Quellen zur Geschichte des NS-Regimes sind unvollständig überliefert und verstreut“. ${ }^{1}$ Davon sind besonders Länder, Reichsgaue, mittlere Reichsbehörden und Mittelinstanzen betroffen, was natürlich direkte Konsequenzen für die Regionalgeschichte hat. Es existieren beispielsweise umfangreiche Akten des „Reichsstatthalters in Bayern und des Bayerischen Staatsministeriums”, während „es von den entsprechenden württembergischen, sächsischen und badischen Behörden nur wenige Aktenreste" gibt. ${ }^{2}$ Oder es fehlen nahzu völlig die Unterlagen der regionalen Dienststellen der Hitler-Jugend und der NS-Frauenschaft. Auch die Akten der Gestapo sind weitgehend vernichtet, 3 wobei Düsseldorf und Würzburg wenige Ausnahmen bilden und dementsprechend von Reinhard Mann und Robert Gellately in prominenter Weise ausgewertet wurden. ${ }^{4} \mathrm{Zu}$ den Problemen der Unvollständigkeit und der Selektivität der Quellen kommt natürlich auch die „Indikatorenqualitat” - also die äußerst problematische Frage nach der Objektivität der Quellen und wie man mit diesen Quellen umgeht. ${ }^{5}$ Für die Zeit der nationalsozialistischen Diktatur ist dies von besonderer Brisanz, weil beispielsweise Gestapo-Berichte, SD-Berichte, Sopade-Berichte (sozialische Geheimbereichte aus Deutschland für die sozialistischen Exilgruppen) oder Quellen über den Holocaust ganz besondere Schwierigkeiten aufwerfen. ${ }^{6}$ Kurzum, Quellenlage und die vorhandenen Quellengattungen konfrontieren Forscher mit erheblichen Problemen.

Eine entscheidende Aufgabe der Forschung ist es, die vorhandenen Quellen in vollem Umfang zu bearbeiten und Wissenslücken zu füllen - und dies ist selbst fast 70 Jahre nach Ende der NS-Diktatur immer noch schwierig genug. Drei Beispiele dazu: Vor kurzem resümierte ein führender Experte über den NS-Genozid, dass „trotz einer Fülle von Darstellungen und Quelleneditionen wichtige Überlieferungen von der Forschung bisher erst ansatzweise aufgearbeitet wurden" - dazu gehören auch die Konzentrationslager und ihre 
Außenkommandos im lokalen und regionalen Bereich. ${ }^{7}$ Gleichfalls kann man feststellen, dass trotz einiger vorzüglicher Arbeiten, überwiegend entstanden seit den 1990er Jahren, unser Wissen über den Antisemitismus vor Ort und in der Region sehr lückenhaft geblieben ist. ${ }^{8}$ Schließlich ist es erstaunlich, dass sich gerade die Regionalforschung weiterhin so wenig systematisch mit visuellen und kartographischen Dokumenten beschäftigt, obwohl beispielsweise vor fast zehn Jahren die Publikation „Fotodokumente des nationalsozialistischen Terrors in der Provinz" grosses Aufsehen erregte und von Forschern als wichtige neue Quelle gepriesen wurde. ${ }^{9}$

Teilweise kommt es immer noch zu erstaunlichen „Neuentdeckungen”, gerade im biographischen Bereich, die neue Einblicke erlauben. Die gerade veröffentlichten Tagebücher des Justizinspektors Friedrich Kellner, der dem Nationalsozialisms ablehnend gegenüberstand, für die Zeit zwischen September 1938 bis zum Kriegsende sind ein gutes Beispiel dafür. ${ }^{10}$ Kellners' Aufzeichnungen sind eine wertvolle Quelle über das Leben im Dritten Reich und das allgemeine Kriegsgeschehen. Aber auch von lokalen Ereignissen und vor allem der Haltung und Reaktion der Bürger in Lauterbach und des Kreises Gießen gegenüber der NS-Propaganda sind sie ein beredtes Zeugnis. Sie geben zudem wichtige Aufschlüsse darüber, was die Bevölkerung über die Ermordung der Juden oder über die Euthanisie-Morde in Deutschland wissen konnte oder sollte. Die Tagebücher machen examplarisch deutlich, „auf welche Weise die nationalsozialistische Propaganda von einem Zeitgenossen dechiffriert werden konnte" und wie weit Wissen über Massenmorde und Gräueltaten im Osten von der Front heimkehrender Soldaten verbreitet war. ${ }^{11}$ Dies galt auch für die Tötungsaktionen in Heil- und Pflegeanstalten. Von besonderer Bedeutung war hier die relativ nahe gelegene Landesheilanstalt Hadamar, die infolge der sogennanten „T4Aktion“ als eine Tötungsanstalt eingesetzt wurde. In ihr wurden insgesamt circa 14.500 sogenannte "Behinderte" ermordet.

Kellners Tägebücher - und Ähnliches könnte man natürlich auch über die von Victor Klemperer aus Dresden sagen ${ }^{12}$ - eröffnen spannende Möglichkeiten für das Verquicken von 
Biographie und einer modernen Regionalgeschichte. Hier ist ein Mann, der vor und nach dem Krieg im regionalen sozialdemokratischen Milieu eingebettet war und deshalb nach 1933 von den hiesigen Nationalsozialisten besonders schikaniert wurde. Letztere versuchten vergeblich, Kellner ins Konzentrationslager Osthofen einzuliefern. Dieser Versuch wurde von der Gießener Kreisleitung abgelehnt, weil ihm dienstlich nichts vorzuwerfen war. NonKonformität, wie beispielsweise der Nichteintritt in die NSDAP, war also möglich, auch wenn dies für Kellner zur Folge hatte, dass er während der Diktatur nicht wieder befördert wurde. Allerdings machen die Tagebücher auch deutlich, dass offene Kritik äußerst gefährlich war. Zudem erlaubt die Auseinandersetzung mit Kellner Hinweise darüber, wieso es nach Kriegsende nicht zu der geplanten „Generalabrechnung” mit den lokalen Nationalsozialisten kam: Kellner war es wohl leid, ein Außenseiter zu sein. Für den Aufbau der Demokratie wurde des Weiteren die Kooperation selbst von ehemaligen Nationalsozialisten benötigt und nicht zuletzt die von oben oktroyierte Entnazifizierungspolitik oftmals als widersprüchlich und ungerecht empfunden.

In der „neuen Täterforschung“, auf die später noch näher eingegangen wird, hat die Verquickung von biographischen Studien mit einem regionalgeschichtlichem Ansatz einen systematischen Einblick in NS-Elite und Herrschaftsstrukturen in Regionen des Deutschen Reiches erlaubt - zum Beispiel in Bayern, Baden, Württemberg, dem Saarland, Lippe und Schleswig-Holstein. Dies führte zur Betonung wichtiger regionaler Besonderheiten. ${ }^{13}$ Solche auf regionalgeschichtlichen Befunden basierenden Kollektivbiographien könnten regionale Vergleiche ermöglichen. ${ }^{14}$ Bislang gibt es diese allerdings nicht, oder lediglich in Ansätzen. Gleichzeitig ist die Biographieforschung, gerade auch in Verbindung mit der Regionalgeschichte, als wertvolle Ergänzung zur Organisations- und Institutionenforschung anzusehen, weil „durch die Auswertung biographischer Zeugnisse [...] der Einfluss von Menschen auf Entwicklung, Wandel und Praxis von Institutionen mit Rücksicht auf ihre biographische Handlungsorientierung untersucht werden“ kann. ${ }^{15}$ 
Forschung widmet diesem Aspekt weiterhin erstaunlich wenig Aufmerksamkeit. Aber auch hier gibt es Ausnahmen, wie Peter Fritzsches gerade publizierte Untersuchung über den „Normalbürger“ Franz Göll, der zwischen 1899 und 1984 in einem Berliner Bezirk lebte und ab 1916 ein Tagebuch führte. ${ }^{16}$ Leider enthält die Studie kaum Informationen über den Kiez und seine Rolle im Leben von Göll. Hier hätte man aufschlussreiche Erkenntnisse über die Spannungen zwischen sozialer Milieus oder traditionellen Institutionen wie Familie, Beruf oder Religion auf der einen Seite, und andererseits der Selbsterkenntnis des Tagebuchschreibers, das Leben in die eigene Hand zu nehmen, gewinnen können. ${ }^{17}$ Beeindruckend ist allerdings, wie Fritzsche deutlich macht, dass Gölls Leben weder in eine Generationstypologie hineingepresst werden kann, noch dem Bild eines „typischen Deutschen" nach dem Ersten Weltkrieg entsprach. Göll hatte wenig über den Versailler Vertrag zu sagen und war nach anfänglicher Begeisterung ein ausgesprochener Gegner der Nationalsozialisten. Hier handelt es sich also um eine Person, die ständig politische Pendelbewegungen mitmachte: Von der SPD wechselt er zur NSDAP, später wird er sich als Gegner des NS-Regimes positionieren, um dann schließlich in Westdeutschland wieder zurück zur SPD unter der Führung Willy Brandt‘s zu kehren. ${ }^{18}$

Oftmals sind chronischeQuellenprobleme für unsere begrenzten Kenntnisse über die sogenannten „kleinen Leute“ verantwortlich. Bislang gibt es kaum Studien, „die sich explizit und methodisch kontrolliert mit den Beweggründen und Handlungsweisen der unteren Funtionäre zwischen 1933 und 1945 auseinandersetzen. “19 Es existiert auch für keine der ungefähr 30.000 NSDAP-Ortsgruppen ein geschlossener Quellenbestand, sondern nur bedingt aussagekräftige Aktenfragmente. ${ }^{20}$ Für ein vollständigeres Bild der Gesellschaft erscheinen Untersuchungen in diesem Bereich jedoch unabdingbar. Gerade der prägende Einfluss von Regionen und Milieus auf den Einzelnen versprechen interessante Erkentnisse. Weiterhin gilt es nicht nur zu fragen, inwieweit Regionen und Räume Menschen prägen, sondern auch inwiefern Menschen diese Räume selber prägen und definieren - aber auch ob und wie sehr sich diese Räume wandeln, da sie „menschlich produziert, gedeutet und ausgehandelt" sind. ${ }^{21}$ Hier gibt es wichtige, aber noch weiterzuentwickelnde Ansätze über 
das zentrale Thema „Heimat und Identität“. Ebenso erscheint es als sinnvoll zu analysieren, inwieweit sich dabei Deutsche inner- und ausserhalb des Reiches gegenseitig beeinflussten. $^{22}$

Ein weiterer wichtiger Quellenfund ist Carsten Schreiber gelungen, der vor wenigen Jahren in einer Außenstelle des Bundesarchives eine Kartei mit biographischen Angaben der geheimen V-Leute und Mitarbeiter des Sicherheitsdienstes im Land Sachsen aufspürte. ${ }^{23}$ Dies ermöglichte Schreiber, erstmals eine detaillierte Untersuchung der regionalen Herrschaftspraxis des Sicherheitsdienstes in einer deutschen Region - am Beispiel Sachsens - durchzuführen. ${ }^{24}$ Die Studie baut auf den Methoden und Ergebnissen der beiden führenden Forschungsrichtungen zum Charakter der nationalsozialistischen Herrschaft auf: Der Denunziationsforschung und der "neuen Täterforschung”. ${ }^{25} \mathrm{Da}$ beide mit der Regionalgeschichte eng verzahnt sind, soll an dieser Stelle näher darauf eingegangen werden.

Die Denunziationsforschung der 1990er Jahre löste die am Leben erhaltene Dichotomie zwischen Herrschern und Beherrschten auf. Die schon erwähnten Gestapo-Untersuchungen von Mann und Gelattely, oder auch Klaus-Michael Mallmanns und Gerhard Pauls Analyse über das Saarland, betonten nun Kollaboration und Konsens der Deutschen während des NS-Regimes. ${ }^{26}$ Plötzlich erschien das Dritte Reich nicht mehr als allmächtiger Überwachungsstaat, sondern als eine Gesellschaft, die ein Regime vor allem von der eigenen Bevölkerung kontrolliert wurde. Insbesondere Angehörige der sozialen Unterschicht machten von der "Waffe” der Denunziation Gebrauch, von der „kleine[n] Macht der Volksgenossen"

Seitdem hat sich das wissenschaftliche Bild einer unwilligen und terrorisierten Bevölkerung stark verschoben hin zu anpassungsfähigen Bürgern, die sich oftmals skrupellos an der 
Raub- und Verfolgungspolitik der Nationalsozialisten beteiligten. Frank Bajohr zeigte dies in seiner 1997 veröffentlichen Studie am Beispiel von Hamburg. In der Hafenstadt allein hatten sich schätzungsweise rund 100.000 Personen aus allen Schichten der Bevölkerung an den öffentlichen Versteigerungen von „billigem“ jüdischen Besitz beteiligt. ${ }^{28}$ Die Hinwendung zu mikro- und alltagsgeschichtlichen Fragestellungen in der Geschichtswissenschaft und das verstärkte Interresse an den Opfern des NS-Regimes hatte seit den 1980er Jahren einen Boom an Lokalgeschichten und von Oral-History-Projekten ausgelöst. Im Bereich der Zwangsarbeit, dem inzwischen wohl am intensivsten erforschten Bereich der wirtschafts-, sozial-, alltags- oder erfahrungsgeschichtlichen NS-Forschung, führte vor allem die Entschädigungsdiskussionen seit Ende der 1990er Jahre zu einer großen Anzahl von lokalund regionalgeschichtlichen Publikationen. Unter anderem wurden Historiker von Kommunen und Landesregierungen beauftragt, einschlägige Quellen zu sichten und Untersuchungen anzustrengen. ${ }^{29}$ Wichtige Bereiche der NS-Zeit blieben aber weiterhin von der Aktensperre betroffen. Dies trifft vor allem auf die Akten des Reichsfinanzministeriums zu - und speziell auf die „Arisierungsakten” der verschiedenen regionalen Oberfinanzdirektionen. Hier haben einige Forscher erfolgreich gegen Bürokratie und Vergangenheitsvertuschung angekämpft und Zugang zu Akten gewonnen, die die massenhafte und weitverbreitete Plünderung jüdischen Eigentums dokumentieren. ${ }^{30}$

Gerade im lokalen und regionalen Bereich ist die nationalsozialistische Vergangenheit immer wieder präsent. Im April 2011 druckte die „Berliner Zeitung“ unter dem Titel „Gute Geschäfte bis zuletzt" eine Rezension einer Ausstellung des Aktiven Museums. Die Ausstellung dokumentiert, wie Berliner Kunsthändler in der NS-Zeit von der Ausplünderung und Ermordung der Juden profitierten. ${ }^{31}$ Der Artikel führt an:

„Während in Stalingrad die Soldaten für Hitlers Eroberungswahn starben, kauften die Volksgenossen an der Heimatfront Gemälde und wertvolle Antiquitäten. Vor dem Auktionshaus von Hans W. Lange in der Berliner Bellevuestraße standen im Januar 1943 elegant gekleidete Menschen Schlange, und der Sicherheitsdienst der SS 
ärgerte sich über die "reichen Globetrotter", die mit Kunstkäufen ihr Vermögen retten wollten. Die Waren waren knapp in der Kriegswirtschaft, wer Geld hatte, versuchte es in krisensicheren Werten anzulegen [...] Wie in allen Lebensbereichen grenzte das NS-Regime auch hier die Juden konsequent aus, beraubte sie ihrer Bürgerrechte und ihrer wirtschaftlichen Existenz, plünderte sie vor der Emigration mit der "Reichsfluchtsteuer" und anderen zynischen Abgaben aus. Die letzte Stufe war der Abtransport in die Lager. Die Ausstellung bietet erschütternde Beispiele. “32

Mehrere Dinge sind hier bemerkenswert: In ehrenamtlicher Arbeit hat ein Verein den Kunsthandel in der Reichshauptstadt zwischen 1933 und 1945 erforscht. In der Tat, Forscher, die nicht hauptberuflich Historiker sind, haben gerade in der Regional- und Lokalgeschichte immer wieder wichtige Impulse gesetzt. ${ }^{33}$ Wenn möglich, sollten diese freiberuflichen Historiker, oder „Hobby-Historiker“, mit Berufshistorikern und Studierenden zusammengebracht werden. Zweitens: Gerade im lokalen- und regionalen Bereich sind natürlich nicht nur detaillierte Mongraphien, sondern auch Ausstellungen und andere praxisnahe und praxis-orientierte Veranstaltungen, Führungen, Publikationen und Mediaereignisse wichtig. Nur eine engagierte, benutzerfreundliche und aktuelle Auseinandersetzung mit der Geschichte vor Ort und in der Region - und ihre Verbindung mit der Gegenwart - eröffnet die Möglichkeit, auch in Zukunft das zentrale Thema „Nationalsozialismus“ für breite Kreise relevant zu machen. Forschung allein und isoliert darf nicht sein. Forscher sollten sich auch mit aktueller Lehr- und Museumspädagogik, mit den Medien und modernen Kommunikationsmöglichkeiten befassen. ${ }^{34}$ Schließlich wird deutlich, dass das Dritte Reich und sein Vermächtnis weiterhin brisant bleiben. Geschichte in der Region hat maßgeblich zur Aufarbeitung einer verdrängten Vergangenheit beigetragen. Inzwischen gibt es unzählige Beispiele, wie man sich in Städten und Dörfern in vielen Teilen Deutschlands damit auseinandersetzt - oder diesen Aufarbeitungsprozess eben blockiert. ${ }^{35}$ Wissenschaftler haben bei der Aufarbeitung von ehemaligen Konzentrationslagern und der Errichtung von Gedenkstätten gerade seit der 90er Jahre wichtige Pionierarbeit geleistet. ${ }^{36}$ Und gerade weil Orte des Terrors, die nun Orte des Erinnerns geworden sind, im Lokalen 
oder Regionalen zu finden sind, hat dieser Bereich auch den Memory-Diskurs entscheidend gefördert.. ${ }^{37}$ Kurzum, vor allem im lokalen und regionalen Bereich scheint es nötig, dass sich die Wissenschaft der zentralen Aufgabe des Memory-Diskurses und der Vergangenheitsbewältigung stellt. Malte Thiessen fasst überzeugend zusammen:

\begin{abstract}
„Obgleich immer häufiger von einer Europäisierung oder gar Globalisierung des kollektiven Gedächtnisses die Rede ist, bleiben selbst transnationale Studien an Erinnerungsgemeinschaften oder -räume gebunden. [...] Konkrete Akteure, Gruppen, Institutionen und Parteien sowie Anlässe, Motive und Hintergründe erlauben einen tiefen Einblick in das "Making History“ von Erinnerungsgemeinschaften. Erst eine Lokalisierung des Gedenkens gibt der Erinnerungskultur die notwendige Bodenhaftung, sodass, wenn man in dem Bild bleiben möchte, der Humus erkennbar wird, auf dem kollektive Narrative entstehen und sich wandeln. “38
\end{abstract}

Unabdingbar dafür ist eine seriöse und differenzierte Forschung, so wie sich etwa Malte Thiessens oder Neil Gregor mit Hamburg und Nürnberg als „Erinnerungsorte” auseinandergesetzt haben. ${ }^{39}$ Leider finden bis heute, vor allem im lokalen und regionalen Bereich, Entlastungsnarrative sowie Helden- und Opfer-Narrative weiterhin weite Verbreitung, inklusive einer Flut von Publikationen, die sich mit der Zerstörung von deutschen Städten während des Luftkrieges beschäftigen. ${ }^{40}$

Inzwischen wissen wir, dass sich nicht nur Privatpersonen, sondern auch Kommunen als „skrupellose Profiteure“ erwiesen und sich die gesamte Bandbreite des städtischen Verwaltungsapparates an den Diskriminierungs- und Verfolgungsmaßnahmen gegen die Juden beteiligten und oftmals in Eigeninitiative vorantrieben. ${ }^{41}$ Ein weiterer Mythos wurde entlarvt: Die angeblich unpolitische und scheinbar machtlose kommunale Verwaltung 
arbeitete in Wirklichkeit Hand-in-Hand mit dem kommunalen NS-Parteiapparat, angetrieben von traditionellem Beamteneifer und Disziplinierungsdruck. Damit trug sie entscheidend zur enormen Dynamik und Leistungskraft des NS-Regimes bei.

Teilweise stark unterschiedliche regionale Traditionen schien dies überhaupt nicht zu beeinflussen. Dabei waren noch während der Weimarer Republik besondere soziale, kulturelle, wirtschaftliche und politische Identitäten in den Regionen für die politische Orientierung und Verhaltensweise der lokalen Bevölkerung von entscheidender Bedeutung. ${ }^{42}$ Dies half nachhaltig zu erklären, warum sich die Nationalsozialisten in Hochburgen der Katholiken oder der Arbeiterbewegung bis 1933 sehr schwer taten, während sie in vielen protestantischen und ländlichen Gegenden, wie vor allem in Franken, so schnell die Macht an sich binden konnten. Letzteres hat vor allem Manfred Kittels Langzeitstudie über die Mentalität und den Protestantismus Westmittelfrankens zu erklären vermocht. ${ }^{43}$ Kurzum, die moderne Regionalgeschichte hat, gerade weil kollektive Einstellungen äußerst wichtig für soziales Verhalten und politisches Handeln sind, die „Wichtigkeit von Mentalitäten, aber auch religiöser Orientierung, für das politische Verhalten der Bevölkerung nachgewiesen“. ${ }^{44}$ Der angedeutete Sprung von der Wichtigkeit zur scheinbaren Nichtigkeit von lokalen, sozialen, kulturellen, wirtschaftlichen und politischen Identitäten in den Regionen für die Verhaltensweise der lokalen Bevölkerung vor und nach 1933 muss also erklärt werden.

Natürlich kann man anführen, dass während des NS-Regimes freie Meinungs- und Willensbildung streng verfolgt wurden. Es gab keine gemeinsamen Organisationen und Netzwerke sowie Ideen und Werten kulturelle Komponente von sinngebenden und mentalitätsprägenden Ideen und Werten, die frei existieren konnten. Trotzdem können all diese Faktoren die äußerst komplexe Entwicklungen während der NS-Diktatur nicht in ihrem ganzen Umfang erklären. Die Forschung sucht weiterhin nach noch stichhaltigeren und weitgreifenderen Thesen, die sich zentralen Fragen stellen: Wie genau konnte es zu einer so rapiden Auflösung der Normen der christlich-humanitären Aufklärung und von moralischen 
Hemmungen kommen ${ }^{45}$ Wieso gelang den Nationalsozialisten selbst in Kerngebieten der Arbeiterbewegung, ob Sachsen oder Württemberg, die „Destabilisierung und Zersetzung der über Jahrzehnte gewachsenen Milieubindungen“? ${ }^{46}$ Wieso verhielt sich eine Gesellschaft wie die in Südwestdeutschland, mit seinen besonderen liberal-demokratischen Traditionen, während des NS-Regimes kaum anders als Deutsche in anderen Regionen ${ }^{47}$ Wieso spielte es absolut keine Rolle, woher die große Gruppe der sogenannten „Schützen“ des Genozids kamen, also die Mitglieder der Wehrmacht, Waffen-SS, Ordnungspolizei und SSEinsatzkommandos? Wer immer sie waren und wo immer sie herkamen - ob alt oder jung, arm oder reich, proletarisch oder bürgerlich, protestantisch oder katholisch, ob aus Baden oder dem österreichischen Kärnten: Ihr mörderisches Auftreten und ihre Effizienz waren erschreckend homogen. ${ }^{48}$ Wie kann man die enorme Dynamik und das Mobilisierungs- und Stabilisierungspotential des NS-Regimes erklären? Auf den Punkt gebracht: Wie war es möglich, dass der nationalsozialistische Genozid aus der Mitte der damaligen Gesellschaft hervorkam ${ }^{49}$

Gerade, weil abstrakt Erscheinendes in die unmittelbare Nähe rückt und greifbarer wird, ist der regional-lokale Blickwinkel für ein besseres Verständnis der Verhaltensweise von Menschen unabdingbar. Dabei ist es egal ob es sich dabei um Täter, Zuschauer, Opfer oder eine Kombination derselben handelt. Diese Ebene befindet sich genau am ",Schnittpunkt von Struktur und Erfahrung, von individualisierender Hermeneutik und strukturanalytischen Verfahren' und eröffnet dem Historiker mithin die Möglichkeit zu mehrperspektivischen Einsichten am konkreten Einzelfall". ${ }^{50}$ Trotz bestehender Desiderate hat die Forschung gerade hier in den letzten 20 Jahren weitreichende Ergebnisse geliefert. Im Folgenden soll hier auf eine Reihe perspektivenreiche Ansätze eingegangen werden, die in der Zukunft weiterhin fruchtbar vertieft werden könnten.

Erstens sollte die Gesellschaft möglichst von vielen Perspektiven und damit von einem 
breiten methodischen Spektrum beleuchtet werden. Es geht also darum, Ansätze aus der Sozial-, Wirtschafts- und Kulturgeschichte, der Frauen- und Geschlechtergeschichte und der Emotionen-, Erfahrungs- und Erinnerungsgeschichte zusammenzubringen. Gleichzeitig sollte versucht werden, bestimmte Themenbereiche, wie etwa Aspekte der Mobilisierung und Strategien, die das Leben von Opfern, Widerständlern, Mitläufern usw. nachhaltig beeinflussten, zu untersuchen. Darüber hinaus müsste diese Analyse mit der Dynamik der unterschiedlichen Phasen, wie auch die radikalisierende Kriegsphase, verbunden werden. Weiterhin sind Fachleute gefordert, sich auch ganz anderen Forschungsdisziplinen zu öffnen. Die moderne Täterforschung hat dies teilweise schon richtungsweisend vorgeführt mit der Einbindung von Soziologie, Psychologie, Rechtswissenschaft und Politikwissenschaft. ${ }^{51}$ Schließlich soll an dieser Stelle noch auf ein bisher kaum erwähntes Phänomen hingewiesen werden: Die Disjunktion in der Kommunikation zwischen Historikern in Deutschland und im Ausland. Während heutzutage von einer Globalisierung des wissenschaftlichen Austausches ausgegangen wird, reden deutsche und nordamerikanische Wissenschaftler oft aneinander vorbei. ${ }^{52}$ Trotz des Internets sowie institutioneller und persönlicher Verbindungen lesen sie oft "nicht dieselbe Literatur und gehen mit unterschiedlichen Grundannahmen an ihr gemeinsames Thema heran." 53 In Deutschland arbeiten Forschergruppen und Sonderforschungsbereiche oftmals an riesigen Projekten mit gemeinsamen Fragestellungen und Methoden. Dieser Ansatz birgt natürlich auch Gefahren in sich: So führte die Dominanz des strukturanalytischen Ansatzes in der deutschen Sozialgeschichte einst zur Vernachlässigung von Mentalitäten und religiöser Orientierungen. Dagegen zeichnet sich vor allem die nordamerikanische Vorgehensweise eher durch Ansatzvielfalt, methodischer Erneuerungen und dem Versuch von Synthesen aus. ${ }^{54}$ Und gerade weil man im regionalgeschichtlichen Bereich eine unübersichtliche Dichte von Spezialstudien und teilweise stark unterschiedliche Forschungsansätze findet ist, es bedauerlich, dass es weiterhin nur wenige systematisch vergleichende Zusammenführungen lokal und regional untersuchter Phänomene gibt. ${ }^{55}$ Gerade deutschen Wissenschaftlern scheint sozusagen der Sprung vom Ästchen zurück zum Ast und weiter zum Baum schwer zu fallen. 
Allerdings gibt es Forscher, die zeigen, wie dieser Blick auf das Größere erfolgreich gelingen kann. Hier ist Hans-Christoph Seidels Studie über den Ruhrbergbau im Zweiten Weltkrieg zu nennen. Diese Arbeit setzt neue Akzente, indem sie einen branchengeschichtlichen Zugang verfolgt, was bislang, in bedingtem Maße, lediglich für die Landwirtschaft gemacht wurde. Von dieser Perspektive aus untersucht Seidel in recht origineller Weise Zwangsarbeit im Kontext von allgemeinen Entwicklungen, wie etwa Produktion und Produktionspolitik, dem gesamten Arbeitseinsatz, der Sozialpolitik und der Sozialgeschichte der Zechenbelgeschaften sowie Arbeits- und Sozialbeziehungen auf der betrieblichen Ebene. ${ }^{56}$ Damit zeigt Seidel, wie man „ausgehend von der Frage der Zwangsarbeit [...] eine breiter angelegte (wirtschaftsgeschichtlich informierte) Organisations- und Sozialgeschichte" während des NS-Regimes anlegen kann. Dabei wird der Ruhrbergbau als Segment der deutschen Kriegswirtschaft, als technisch-betrieblicher Zusammenhang und als Akteur in der Arbeitseinsatz-, Sozial- und Wirtschaftspolitik begriffen. Das ermöglicht es, zentrale Fragen der Zwangsarbeiterforschung aufzugreifen.

Zweitens eröffnet das Volksgemeinschafts-Konzept große Chancen für eine vertiefte Auslotung des Verhaltens der deutschen Gesellschaft während des Dritten Reiches. Gerade auf der lokalen Ebene sollten Abwandlungen seiner Bedeutung und seiner Wirkungskraft noch untersucht werden. Vor Ort und in den Regionen lassen sich konkrete Vergemeinschaftsprozesse wohl am besten fassen. Sie eröffnen neue Perspektiven. ${ }^{57}$ Hier lässt sich die unglaubliche Mobilisierung der Deutschen, die Dynamik und Vitalität von freigesetzten Energien, das Gefühl sozialer Gleichheit, die Gemeinschaft und nationale Einheit, konkret beobachten. ${ }^{58}$ Und schließlich kann man hier geradezu hautnah die politische Inklusion und rassistische Exklusion studieren. Es zeigt sich, wie gewöhnliche Deutsche die eskaliendende Judenverfolgung billigten, unterstützen oder aber sich von ihr distanzierten. Schließlich erlaubt diese Perspektive auch Einblicke in die Verhaltensweisen und die Reaktionen der Opfer. ${ }^{59}$ Ziel des Volksgemeinschafts-Konzepts ist es also nicht, Homogenitäten oder Dichotomien in der deutschen Gesellschaft aufzuzeigen. Vielmehr hilft dieser Ansatz zu bestimmen, wie sich Menschen unter bestimmten Bedingungen verhalten 
und dabei diese Bedingungen selber produzieren oder reproduzieren. Hier geht es darum, die variable soziale Praxis von Menschen zu untersuchen - und in den Worten von Michael Wildt „[...]eben um die Vielfältigkeit von Handlungsweisen, von Mit-Tun wie Sich-Abwenden, Bereitwilligkeit wie Widerwille, Anpassungsbereitschaft wie Begeisterung, Sich-Distanzieren wie ‘Dem-Führer-Entgegenarbeiten'”. ${ }^{60}$

Drittens, soll hier jeweils ein richtungsweisendes Beispiel von einem sogenannten „harten” Thema, in diesem Fall geht es um Organisation, Struktur, Funktion und Herrschaftspraxis, und von einem „weichen” Thema, hier geht es um Mentalität und Kultur, beleuchtet werden. Seit einigen Jahren befassen sich vor allem deutsche Forscher mit großem Interesse mit den Funktionsmechanismen und Herrschaftstechniken der NS-Diktatur, um ganz konkret die immer wieder bemerkten Mobilisierungserfolge des Regimes erklären zu können. Dabei sind die regionalen Mittelinstanzen in den Fokus gekommen, weil gerade dort, und ganz im Gegensatz zur Reichsebene, ein hohes $M a \beta$ an Stabilisierungs- und Mobilisierungspotenziale zu finden sind. Markus Fleischhauers richtungsweisende Studie der Struktur- und Funktionsgeschichte des NS-Gaus Thüringen zeigt, wie verfestigte Herrschaftsprinzipienund Mechanismen im Krieg durch engmaschige Beziehungs- und Organisationsgeflechte zwischen Eliten aus Partei, Staat, Wehrmacht und Wirtschaft ergänzt wurden. ${ }^{61}$ Dies führte zu neuen Aktionsräumen und förderte Formen der Kommunikation und Interaktion, was „die erheblichen Mobilisierungs- und Bindekräfte" freisetzte. Voraussetzung dafür war, dass innerhalb der Eliten ein Konsens bestand über „die Durchführung des Krieges, die Aufrechterhaltung der Leistungsfähigkeit der Kriegswirtschaft und des Arbeitseinsatzes und die Stabilität der ,Heimatfront““. ${ }^{62} \mathrm{Im}$ Mittelpunkt dieses dynamischen und hockkommunikativen Herrschaftsapparats im Gau Thüringen stand Gauleiter Fritz Sauckel, der durch seine besondere Durchsetzungsfähig und sein organisatorisches Geschick die politischen Fäden im Gau in seinen Händen hielt.

Netzwerke und Kommunkationsformen, die als sogennante „Schmiermittel stabilisierender und mobilisierender Tendenzen im Nationalsozialismus" diskutiert werden, sind momentan dementsprechend näher in den Fokus gerückt. ${ }^{63}$ Forscher sehen hier eine zentrale 
Erklärung für die „innere Dynamik” des NS-Systems, das entscheidend auf Personalisierung und Improvisation beruhte. ${ }^{64}$ Bei einer Tagung über dieses Thema ist unlängst deutlich geworden, wie viele Aspekte bei diesem komplexen Thema noch immer umstritten sind. Im Verlauf dieser Konferenz wurde eine Vielzahl von erheblichen Problemen aufgeworfen: Es gibt Quellen- und Methodenprobleme bei der Untersuchung informeller Kooperationsformen oder den Intentionen und Strategien der jeweiligen Akteure. Darüber hinaus ist es umstritten, ob man formelle und informelle Kooperationsformen voneinander trennen sollte oder nicht. Der „Mittelinstanz"-Begriff und der Kooperationsbegriff werden als unpräzise kritisiert. Einige Fachleute monieren die Schwierigkeit, zeitliche und geographische Faktoren sowie vertikale und horizontale Dimensionen gleichzeitig zu berücksichtigen. Des Weiteren ist das genaue Verhältnis von Makro-, Meso- und Mikroebenen nicht geklärt. Die Experten sind sich uneinig, ob der Polykratie-Begriff und ihr Modell in der neuesten Forschung von Nutzen sind und weiter benutzt werden sollten. Schliesslich sind systematische Vergleiche des NS- Herrschafts- und Kriegssystems mit anderen modernen Diktaturen weiterhin ein Forschungsdesiderat. ${ }^{65}$

Für andere Forscher geht indes die These einer örtlichen Selbststabilisierung des NS-Staats zu weit. Jörn Brinkhus, der die systemstabilisierende Funktion der Gemeindeverwaltungen und Mittelbehörden im Bereich des zivilen Luftschutzes und der Versorgungspolitik der Bevölkerung mit Konsumgütern des täglichen Bedarfs mit Hilfe von vergleichenden Lokalstudien untersuchte, warnt davor “[...]die stabilisierende Wirkung der Selbstorganisation der Mittel- und Unterbehörden nicht zu hoch zu veranschlagen." ${ }^{66}$ Am Ende kommt er zu einem nüchternen Resümee über die Funktionsweise der NS-Diktatur im Spannungsfeld zwischen Zentrum und Peripherie:

„Auf dem Nährboden der polykratischen und über-zentralisierten Struktur des NSStaats enfalteten sich verschiedene Formen von Selbstorganisation, die von den Entscheidungsträgern vor Ort genutzt wurden, um mit den Steuerungs- und Organisationsproblemen dieses Herrschaftsgefüges umzugehen, ohne freilich eine 
eigenen politische Ordnung zu etablieren. Mit einer Revitalisierung kommunaler Selbstverwaltung oder gar der Wiederbelebung eines Föderalismus hatte diese Form der Selbstorganisation nichts zu tun, war sie doch lediglich eine Reaktion auf die Defizite und Dysfunktionalitäten des Regimes. ${ }^{“ 67}$

Die Forschung benötigt also weitere Untersuchungen, die von anderen Bereichen und Regionen ähnlich tiefe Erkenntnise über die Funktionsweisen und das Organisationsgeflecht der NS-Diktatur vor und während des Krieges liefern. Erst dann wird es möglich sein, die teilweise unterschiedlichen Ergebnisse von Fleischhauer und Brinkhus besser in ein Gesamtbild einzuordnen.

Forschungen mit sogenannten „weichen” kultur- und mentalgeschichtlichen Änsatzen haben seit den 1990er Jahren zu einer Reihe von richtungsweisenden Studien geführt, die das NSHerrschafts- und Terrorsystem direkt in einem spezifischen regionalen Umfeld untersuchen. ${ }^{68}$ Martina Stebers unlängst publizierte Untersuchung über das bayerische Schwaben vom Kaiserreich bis in die NS-Zeit zeigt, wie man weiterhin mit neuen Fragen und Perspektiven originelle Erkenntnisse gewinnen kann. ${ }^{69}$ Steber macht deutlich, wie sich das Regionale in Schwaben seit der Jahrhunderwende immer deutlicher als Ordnungsentwurf und "integrierende Klammer" in einer als krisengeschüttelt wahrgenommenen Gegenwart herauskristallisierte. Während des Dritten Reiches verschwand dann das bislang existierende vormoderne Gesellschaftsbild und das Regionale erhielt, durch die dynamische Neuausrichtung der Gaukulturpolitik, ein genuin nationalsozialistisches Gesicht. Im Gau Schwaben wandelte sich das Regionale also nach 1933 und „entwickelte sich zum kulturellen Resonanzboden des Konzept einer 'völkischen Leistungsgesellschaft'". Steber betont demnach die integrative Funktion des Regionalen. Die gedachte Ordnung des Regionalen wurde in recht eigenwilliger Weise Ausdruck „radikalen Ordnungsdenkens" im NS-Regime. Solch eine Verquickung von Regionalgeschichte mit einem langzeitlichen mental- und kulturgeschichtlichen Ansatz versteht zu überzeugen und kann in den unterschiedlichsten Bereichen ausgelotet werden. Dies versuchen beispielsweise 
Caitlin E. Murdock in ihrer Studie über Tourismus, Landschaft und regionale Identität in Sachsen oder Martin Ulmers mit seiner Untersuchung über den Antisemitismus in Stuttgart. ${ }^{70}$ Außerdem gibt es weiterhin kaum vergleichende Studien mit Regionen außerhalb Deutschlands und Untersuchungen von Grenzregionen sowie deren spezifische I dentitäten. ${ }^{71}$

Untersuchungen über die „Vernichtungspolitik aus lokaler und regionaler Nahperspektive“ haben in den letzten 20 Jahren unser Wissen entscheidend bereichert. Dies gilt für viele Bereiche, inklusive der sogenannten NS-„Euthanasie“, der Geschichte der Konzentrationslager, der Rolle der Frauen beim Massenmord und des Genozids in den besetzten Gebieten. ${ }^{72}$ Auf Letzteren soll hier näher eingegangen werden. Eine neue Generation junger Forscher veröffentlichte seit den 1990er Jahren wichtige Regionalstudien über den Holocaust in den besetzten Gebieten. Sie stützten sich auf umfassende Quellenauswertungen in den neueröffneten Archiven Osteuropas sowie auf bis dahin vernachlässigte deutsche Gerichtsakten. Diese Studien setzten sich direkt mit Tätern und Tatorten, Helfershelfern und Nutznießern, aber auch dem Schicksal von Opfern, auseinander. Sie betonten die Vielfalt der Mordmotive (Rassenwahn, Gruppenzwang, Radikalisierung in einem brutalen Krieg, pervertierter Lust am Töten, persönliche Bereicherung, utilitaristische Motive usw.), regionale Besonderheiten für den Anstoß und die Ausführung zum Massenmord. und Unterschiede. Es wurden aber auch Ähnlichkeiten in den Verhaltensweisen der einheimischen Bevölkerung während des Genozids in den besetzten Gebieten aufgezeigt. Dieser Forschungsbereich muss sich mit erheblichen Problemen auseinandersetzen. Zum einen ist die Verknüpfung von Täter- und Opferperspektive in dem komplexen Geflecht von Besatzungterror, Kollaboration und Judenvernichtung oftmals schwer durchzuführen. ${ }^{73}$ Zum anderen ist die Untersuchung von Gebieten, die nacheinander von Deutschen und Sowjets besetzt wurden, äußerst kompliziert, da sich Forscher unter anderem mit verschiedenen regionalen Traditionen, Sprachen und Herrschaftssystemen 
auseinandersetzen müssen. ${ }^{74}$ Es kann somit konstatiert werden, dass auch hier bei näherem Hinschauen viele Forschungsdesiderata bleiben. Beispielsweise gibt es kaum Arbeiten über die Okkupationspolitik, das Schicksal der Juden in Belgien oder vergleichende Studien über die NS-Okkupation in West- und Osteuropa.

Die moderne und florierende Täterforschung greift vor allem auf Kollektivbiographien zurück: Forscher betrachten Täterkollektive innerhalb von SS, SD, Gestapo, Einsatzkommandos, Ordnungspolizei, NSDAP-Kreise usw. und versuchen, „biographische Knotenpunkte, gemeinsame Sozialisationserfahrungen, Radikalisierungsmomente und gruppendynamische Konditionierungen zu identifizieren". ${ }^{75}$ Diese Forschungsansätze machen deutlich, dass die Beschränkung auf die relative knappe Zeitspanne zwischen den epochalen Eckpunkten 1933 und 1945, oder sogar nur auf die Kriegsphase, wie es in der älteren Forschung oftmals gemacht wurde, nicht ausreicht. Lebensläufe, mentale Prägungen, Milieu-Zusammenhänge und vieles mehr begannen früher, endeten später und können nur mit Focus auf einen längeren Zeitraum untersucht werden. ${ }^{76}$ Das bedeutet auch, dass der „Blick über den Tellerrand der NS-Forschung" gewagt werden sollte, um beispielsweise Vergleiche zwischen der Durchdringung der Gesellschaft mit einem V-Netz im Dritten Reich und der DDR zu machen. ${ }^{77}$ Nur so lassen sich dann auch Vergleiche anstellen und Kontinuitäten sowie Diskontinuitäten aufzeigen, z. B. zwischen der Dichte und dem Personal des SD-Netzes und des IM-Apparats der Staatssicherheit der DDR. ${ }^{78}$ Carsten Schreibers Studie zeigt auf regionaler Ebene zum ersten Mal die tiefe Verankerung des SD in Kultur, Staat, Wirtschaft und Wissenschaft. ${ }^{79}$ Sächsische V-Leute bildeten eine systematische Ergänzung zu den spontanen Denunzianten und den gesteuerten Gestapoagenten. V-Leute waren ehrenamtlich in einem sozialen Netzwerk tätig und mit ihren vielfältigen beruflichen Kontakten und gesellschaftlichen Bindungen lokal fest verankert. Genau an dieser Stelle, der Inkorporierung - also nicht Ersetzung - von Entscheidungsträgern aus der inneren Verwaltung, aus dem staatlichen Gesundheitswesens, der Justiz usw. ist die tiefere Bedeutung des SD für die regionale Herrschaft zu suchen. 
Der Mythos einer harmlosen oder vom Zentrum terrorisierten Provinz oder Region ist längst nicht mehr haltbar. Terror und Vernichtungspolitik hatten ihren Ursprung gerade auch im Lokalen und Regionalen, wo sie praktiziert und von wo aus sie oftmals auch exportiert wurden. Selbst ehrenamtliche sächsische V-Leute wurden wiederholt im Osten als Exekutoren der Vernichtungspolitik eingesetzt und waren Teil einer bislang kaum erforschten Gruppe von Vollstreckern des rassistischen Genozids. Schreiber bilanziert, dass „ihr ständiger Wechsel zwischen Heimatfront und Kommandoeinsatz mit tausendfachem Tötungsauftrag" direkt zu einer brutalisierenden Wirkung ihres Handelns in Sachsen führte. ${ }^{80}$ Der regionalgeschichtliche Ansatz hat Studien über die Haltung und das Verhalten der Bevölkerung zum NS-Regime geprägt und entscheidend weitergeführt. Dies bedeutet jedoch nicht, dass sich die Forschung in zentralen Fragen einig ist. War es nun eine „Konsensdiktatur“, die geprägt war von massenhaftem Mitläufertum, oder spielt solch eine Beschreibung Aspekte des Terrors und des Zwangs herunter? ${ }^{81}$ Wie verbreitet und tiefsitzend war der Antisemitismus oder Rassismus - also Kernelemente der NS-Ideologie in der deutschen Bevölkerung? ${ }^{82}$ Gab es einen wachsenden und weitverbreitenden antisemitischen Konsens (Friedländer; Bajohr/Pohl), oder musste nationalsozialistische Politik gegen die Juden ziemliches Unverständnis, Skepsis und Kritik überwinden (Longerich)? ${ }^{83}$ Was wussten die Deutschen über den Holocaust? ${ }^{84}$ Wodurch wird das Verhalten der „Volksgemeinschaft“ während der NS-Diktatur erklärt? War es eine Mischung von Befehlsgehorsam, eines schleichenden Prozesses der Anpassung und Angewohnheit an extreme Ungleichheiten (Wehler) ${ }^{85}$ oder wandelten sich „normale” Deutsche in pro-aktive Rassisten?

Man sollte nun nicht verlangen, dass die Regionalgeschichte zu all diesen Fragen Antworten liefern kann. Vielmehr sollte man sich in Erinnerung rufen, dass dieser und ihr nahestehende Forschungsansätze ja gerade dadurch überzeugen, dass sie generalisierende Aussagen in Frage stellen! Hier, in der Region und vor Ort, treten auch die Schwierigkeiten 
zu Tage, konkrete Aussagen zu tätigen. Diese sollten zwar nicht nur über eine Person, aber dennoch über einen Teil einer Bevölkerung von fast 80 Millionen Menschen, inklusive Österreich und dem Sudentenland, mit all ihren komplexen Identitäten - und das während einer brutalen Diktatur - getätigt werden. Zudem betont die heutige Forschung insbesondere die Komplexität, den wechselseitigen Einfluss von Zentrum und Peripherie, von Langzeitfaktoren oder situationsbedingten Faktoren, von Zeit und Ort sowie vielen anderen Zwischenzonen und Grauzonen. Dabei muss das genaue Verhältnis und die Interaktion zwischen unzähligen Faktoren und handelnden Menschen immer wieder neu durchdacht und bestimmt werden. Dass dies nicht immer zu schlüssigen Erklärungen führen kann oder dies manchmal mehr Fragen aufwirft als man Antworten liefern kann, sollte öfters und klarer deutlich gemacht werden. Darüber hinaus muss man akzeptieren, auch Zweideutigkeiten und Ambivalenzen aufzudecken. Vor kurzem bilanzierte Peter Fritzsche zu Recht, dass die Forschung weiterhin nach Möglichkeiten suchen muss, wie man das Hin und Her auslotet zwischen der ideologischen Vision Hitlers, der lokalen Massenpraxis der Gewalt - ob in der Hauptstadt oder an den Kriegsschauplätzen - und der individuellen Entscheidung, wie man sein Handeln selbst ausgestaltet. Denn letztendlich hatte jeder Deutsche die Option, zuzuschauen, teilzunehmen, sich durchzumogeln, zu widerstehen oder eine Kombination von diesen Verhaltensweisen. ${ }^{86}$

\footnotetext{
${ }^{1}$ Heinz Boberach, Quellen zum Nationalsozialismus. In: Wolfgang Benz/Hermann Graml/Hermann Weiß (Hg.), Enzyklopädie des Nationalsozialismus, Stuttgart 1997, S. 330341, hier 330.

2 Ebd.

${ }^{3}$ Ebd.

${ }^{4}$ Reinhard Mann, Protest und Kontrolle im Dritten Reich. Nationalsozialistische Herrschaft im Alltag einer rheinischen Großstadt, Frankfurt a. M. 1987; Robert Gellately, The Gestapo and German Society. Enforcing Racial Policy, 1933-1945, Oxford 1990.
} 
${ }^{5}$ Carsten Schreiber, Elite im Verborgenen. Ideologie und regionale Herrschaftspraxis des Sicherheitsdienstes der SS uns sines Netzwerks am Beispiel Sachsens, München 2008, S. $308 f$.

${ }^{6} \mathrm{Vgl}$. die Diskussionen über den Qellenumgang in den Vorworten zu folgenden Publikationen: Bernd Stöver, Volksgemeinschaft im Dritten Reich. Die Konsensbereitschaft der Deutschen aus der Sicht sozialistischer Exilberichte, Düsseldorf 1993; Wolfgang Ribbe, (Hg.), Die Lageberichte der Geheimen Staatspolizei über die Provinz Brandenburg und die Reichshauptstadt Berlin: 1933 bis 1936. Boehlau, Koeln. Band. 1. Der Regierungsbezirk Potsdam, eingeleitet von Sibylle Hinze, Köln 1998; Meldungen aus dem Reich. Die geheimen Lageberichte des Sicherheitsdienstes der SS 1938-1945. 17 Bände, Herrsching 1984; Otto D. Kulka/Eberhard Jäckel (Hg.), Die Juden in den geheimen NS-Stimmungsberichten 19331945, Düsseldorf 2004; Raul Hilberg, Die Quellen des Holocaust entschlüsseln und interpretieren, Frankfurt a. M. 2002.

${ }^{7}$ Jürgen Matthäus, Quellen. In: Wolfgang Benz/Barbara Distel (Hg.), Der Ort des Terrors. Geschichte der nationlsozialistischen Konzentrationslager, Band 1, München 2005, S. 363376, hier 363.

${ }^{8}$ Zwei neue Studien in diesem Bereich sind: Martin Ulmer, Antisemitismus in Stuttgart 1871-1933: Studien zum öffentlichen Diskurs und Alltag, Stuttgart 2011; Hannes Heer/J ürgen Kesting/Peter Schmidt (Hg.), Verstummte Stimmen. Die Vertreibung der "Juden" und "politisch Untragbaren" aus den hessischen Theatern 1933 bis 1945, Berlin 2011.

${ }^{9}$ Klaus Hesse/Philip Springer, Vor aller Augen. Fotodokumente des nationalsozialistischen Terrors in der Provinz, Essen 2002. Vgl. auch Cécile Desprairies, Sous I'oeil de l'occupant. La France vue par l'Allemagne 1940-1944, Paris 2010; X Riss, Hitler dans mon salon, Paris 2009

${ }^{10}$ Friedrich Kellner, »Vernebelt, verdunkelt sind alle Hirne«: Tagebücher 1939-1945, 2 Bd., Göttingen 2011. Vgl. auch: Markus Roth, Chronist der Verblendung - Friedrich Kellners Tagebücher 1938/39 bis 1945. Beiheft zur Ausstellung: Die Last der ungesagten Worte. 
Gesprächskreis Geschichte Heft 83. URL: library.fes.de/pdf-files/historiker/06884.pdf. Abgerufen am 13.4.2012.

${ }^{11}$ Kellner, Tagebücher 1939-1945, S. 10, 1104.

${ }^{12}$ Victor Klemperer, Ich will Zeugnis ablegen bis zum letzten. Tagebücher. Bd. 1: 19331941, Bd. 2: 1942-1945, Berlin 1995.

13 Sebastian Lehmann, Kreisleiter der NSDAP in Schleswig-Holstein. Lebensläufe und Herrschaftspraxis einer regionalen Machtelite, Bielefeld 2007; Michael Kißener/Joachim Scholtyseck (Hg.), Die Führer der Provinz. NS-Biographien aus Baden und Württemberg, Konstanz 1999; Christine Arbogast, Herrschaftsinstanzen der württembergischen NSDAP. Funktion, Sozialprofil und Lebenswege einer regionalen NS-Elite 1920-1960, München 1998; Andreas Ruppert/Hansjörg Riechert (Hg.), Herrschaft und Akzeptanz. Der Nationalsozialismus in Lippe während der Kriegsjahre. Analyse und Dokumentation, Opladen 1998; Gerhard Paul, Ganz normale Akademiker. Eine Fallstudie zur regionalen staatspolizeilichen Funktionselite. In: Sebastian Lehmann/Klaus-Michael Mallmann (Hg.), Die Gestapo - Mythos und Realität, Darmstadt 1995, S. 236-254.

${ }^{14}$ Tagber: Die biographische Methode in der Regionalgeschichte. LWL-Institut für westfälische Regionalgeschichte 25.09.2009, Münster. URL: http://hsozkult.geschichte.huberlin.de/tagungsberichte/id=2942. Abgerufen am 13.4.2012.

${ }^{15}$ Ebd.

${ }^{16}$ Peter Fritzsche, The Turbulent World of Franz Göll. An ordinary Berliner Writes the Twentieth Century, London 2011.

${ }^{17}$ Ebd., S. 10.

${ }^{18}$ Ebd., S. 7. Siehe auch die Rezension von Benjamin Ziemann, URL: http://hsozkult. geschichte.hu-berlin. de/rezensionen/2011-4-048. Abgerufen am 13.4.2012. ${ }^{19}$ Christine Müller-Botsch, "Den richtigen Mann an die richtige Stelle." Biographien und politisches Handeln von unteren NSDAP-Funktionären, Frankfurt a. M. 2009, S. 9.

${ }^{20}$ Carl-Wilhelm Reibel, Die NSDAP-Ortsgruppen Dornbusch und Oberrad 1933-1945. In: Dieter Rebentisch (Hg.), Die Herrschaft des Nationalsozialismus in Frankfurt am Main, 
Frankfurt a. M. 1999, S. 53-120, hier 53. Siehe auch Carl-Wilhelm Reibel, Das Fundament der Diktatur. Die NSDAP-Ortsgruppen 1932-1945, Paderborn 2002; Für eine Analyse von Stuttgarter NSDAP-Funktionären siehe Müller-Botsch, “Den richtigen Mann”.

${ }^{21}$ Tagber: Die biographische Methode.

${ }^{22}$ Krista O’Donnell/Renate Bridenthal/Nancy R. Reagin (Hg.), The Heimat Abroad. The Boundaries of Germanness, Ann Arbor 2005. Augenblicklich in Druck: Claus-Christian W. Szejnmann/Maiken Umbach (Hg.), Heimat, Region and Empire. Spatial Identities under National Socialism, Basingstoke 2012.

${ }^{23}$ Schreiber, Elite im Verborgenen.

${ }^{24}$ Ebd., S. 3.

${ }^{25}$ Ebd., S. 7.

${ }^{26}$ Mann, Protest und Kontrolle; Gellately, Gestapo and German Society; Klaus-Michael Mallmann/Gerhard Paul/Hans-Walter Herrmann, Widerstand und Verweigerung im Saarland 1935 bis 1945. Bd. 1: Das zersplitterte Nein, Bonn 1989; Bd. 2: Herrschaft und Alltag: ein Industrierevier im Dritten Reich, Bonn 1991; Bd. 3: Milieus und Widerstand Bonn, 1995. ${ }^{27}$ Schreiber, Elite im Verborgenen, S. 11.

${ }^{28}$ Frank Bajohr, „Arisierung“ in Hamburg. Die Verdrängung der jüdischen Unternehmer 1933-45, Hamburg 1997. Vgl. auch Raubgut. Interview Frank Bajohr. Art. Das Kunstmagazin. 1.2.2011. URL: http://www.artmagazin.de/szene/38495/interview_frank_bajohr_raubgut. Abgerufen am 13.4.2012. ${ }^{29}$ Hans-Christoph Seidel, Der Ruhrbergbau im Zweiten Weltkrieg. Zechen - Bergarbeiter Zwangsarbeiter, Essen 2010, S. 1f.

${ }^{30}$ Vgl. das Interview mit Wolfgang Dreßen in der taz: Gabriele Goettle, Plünderung jüdischen Eigentums. Billigende Inkaufnahme. "Wie Deutsche ihre jüdischen Mitbürger verwerteten". In: taz, 28.11.2010; Vgl. auch Legalisierter Raub. Der Fiskus und die Ausplünderung der Juden in Hessen 1933-1945. Katalog zur Ausstellung. Redaktion: Katharina Stengel, Susanne Meinl, Bettina Leder-Hindemith. Mitarbeit: Nassrin Sadeghi, Birgit Körner, Stephan Wirtz. Gestaltung: Madlen Mühlender. Reihe selecta der 
Sparkassen-Kulturstiftung Hessen-Thüringen, Heft 8, 3. Auflage 2008; Katharina Stengel (Hg.), Vor der Vernichtung Die staatliche Enteignung der Juden im Nationalsozialismus, Frankfurt a. M. 2007; Susanne Meinl/Jutta Zwilling, Legalisierter Raub. Die Ausplünderung der Juden im Nationalsozialismus durch die Reichsfinanzverwaltung in Hessen, Frankfurt a. M. 2004.

${ }^{31}$ Sebastian Preuss, Gute Geschäfte bis zuletzt. In: Berliner Zeitung, 20.4.2011. URL: http:// www. berliner-zeitung. de/archiv/ eine-ausstellung-des-aktiven-museums-zeigt-- wieberliner-kunsthaendler-in-der-ns-zeit-von-der-auspluenderung-und-ermordung-der-judenprofitierten-gute-geschaefte-bis-zuletzt,10810590,10783104.html. Abgerufen am 13.4.2012. 32 Ebd.

${ }^{33}$ Ernst Klee, „Euthanasie“ im NS-Staat: Die „Vernichtung lebensunwerten Lebens“, Frankfurt a. M. 1983; Götz Aly/Susanne Heim, Vordenker der Vernichtung. Auschwitz und die deutschen Pläne für eine neue europäische Ordnung, Hamburg 1991.

${ }^{34}$ Vgl. Beispielsweise die Forschungsgruppe Innerdeutsche Grenze, "Grenzerfahrungen”. URL: http://www.grenzprojekt.de/innerdeutsche-grenze.html. Abgerufen am 13.4.2012. ${ }^{35}$ Harald Welzer (Hg.), Der Krieg der Erinnerung. Holocaust, Kollaboration und Widerstand im europäischen Gedächtnis, Frankfurt a. M. 2007; Peter Schyga, Goslar 1918-1945. Von der nationalen Stadt zur Reichsbauernstadt des Nationalsozialismus, Bielefeld 1999, S. 22, 40. Anna E. Sosmus, Widerstand und Verfolgung am Beispiel Passaus 1933-1939, Passau 1983 (vgl. Michael Verhoeven's Film ‘Das schreckliche Mädchen’/The Nasty Girl von 1990. ${ }^{36}$ Vgl. Beispielsweise Rainer Schulze und Wilfried Wiedemann (Hrsg.), AugenZeugen: Fotos, Filme und Zeitzeugenberichte in der neuen Dauerausstellung der Gedenkstätte BergenBelsen. Hintergund und Kontext, Celle 2007; Winfrid Nerdinger, Ort und Erinnerung: Nationalsozialismus in München, Salzburg 2006; Carina Baganz, Erziehung zur „Volksgemeinschaft“? Die frühen Konzentrationslager in Sachsen 1933-1934/37, Berlin 2005; Jan Erik Schulte (Hrsg.), Konzentrationslager im Rheinland und Westfalen 1933-1945, Paderborn 2005. Vgl. auch folgendes Standardwerk: Ulrike Puvogel/Martin Stankowski (Hg.), Gedenkstätten für die Opfer des Nationalsozialismus. Eine Dokumentation. 2., 
überarbeitete und erweiterte Auflage, 2 Bd., Bonn 1996/1999. Für Studien über den nationalen Memory-Kurs siehe Norbert Frei/J osé Brunner/Constantin Goschler (Hg.), Die Praxis der Wiedergutmachung. Geschichte, Erfahrung und Wirkung in Deutschland und Israel, Bonn 2010; Peter Reichel/Harald Schmid/Peter Steinbach (Hg.), Der Nationalsozialismus. Die zweite Geschichte. Überwindung - Deutung - Erinnerung, Bonn 2009; Richard Ned Lebow/Wulf Kansteiner/Claudio Fogu (Hg.), The Politics of Memory in Postwar Europe, London 2006; Volkhard Knigge/Norbert Frei (Hg.), Verbrechen erinnern. Die Auseinandersetzung mit Holocaust und Völkermord, Bonn 2005.

${ }^{37}$ Annette Kaminsky (Hg.), Orte des Erinnerns. Gedenkzeichen, Gedenkstätten und Museen zur Diktatur in SBZ und DDR, Bonn 2004; Ulrike Puvogel/Martin Stankowski (Hg.), Gedenkstätten für die Opfer des Nationalsozialismus. Eine Dokumentation. 2., überarbeitete und erweiterte Auflage, 2 Bd., Bonn 1996/1999.

${ }^{38}$ Malte Thiessen, Hamburgs Gedenken an Luftkrieg und Kriegsende 1943 bis 2005, Hamburg 2007, S. 461.

${ }^{39}$ Neil Gregor, Haunted City. Nuremberg and the Nazi Past, London 2007; Thiessen, Hamburgs Gedenken an Luftkrieg und Kriegsende.

${ }^{40}$ Vgl. beispielsweise Egbert A. Hoffmann, Als der Feuertod vom Himmel stürzte. Hamburg Sommer 1943, Gudensberg-Gleichen 2003; Christian Hanke/J oachim Paschen/Bernhard J ungwirth (Hg.), Hamburg im Bombenkrieg 1940-1945. Schicksal einer Stadt, Hamburg 2003.

${ }^{41}$ Sabine Mecking/Andreas Wirsching, Stadtverwaltung als Systemstabilisierung? Tätigkeitsfelder und Handlungsspielräume kommunaler Herrschaft im Nationalsozialismus. In: dies. (Hg.), Stadtverwaltung im Nationalsozialismus. Systemstabilisierende Dimensionen kommunaler Herrschaft. Paderborn 2005, S. 1-19.

${ }^{42}$ Claus-Christian W. Szejnmann, Theoretisch-methodische Chancen und Probleme regionalgeschichtlicher Forschungen zur NS-Zeit. In: Michael Ruck/Karl Heinz Pohl (Hg.), Regionen im Nationalsozialismus, Bielefeld 2003, S. 43-57. 
${ }^{43}$ Manfred Kittel, Provinz zwischen Reich und Republik. Politische Mentalitäten in Deutschland und Frankreich 1918-1933/36, München 2000.

${ }^{44}$ Claus-Christian W. Szejnmann, Die Bedeutung der Regionalgeschichte für die Erforschung des Nationalsozialismus und des Holocausts. In: Olaf Hartung/Katja Köhr (Hg.), Geschichte und Geschichtsvermittlung. Festschrift für Karl Heinrich Pohl, Bielefeld 2008, S. 85-103, hier 87. Vgl. auch Claus-Christian W. Szejnmann, Verwässerung oder Systemstabilisierung? Nationalsozialismus in Regionen des Deutschen Reiches. In: Neue Politische Literatur, 48 (2) (2003), S. 208-250.

${ }^{45}$ Hans-Ulrich Wehler, Deutsche Gesellschaftsgeschichte 1914-1949, München 2003, S. 687. ${ }^{46}$ Claus-Christian W. Szejnmann, Arbeitermilieus in Südwestdeutschland in der Auseinandersetzung mit dem Nationalsozialismus. In: Peter I. Trummer/Konrad Pflug (Hg.), Die Brüder Stauffenberg und der Deutsche Widerstand, Stuttgart 2006, S. 51-64, hier 64. ${ }^{47}$ Ebd.

${ }^{48}$ Vgl. George C. Browder, Perpetrator Character and Motivation: an Emerging Consensus? In: Holocaust and Genocide Studies, 17 (2003) 3, S. 480-497, hier 481. Zitiert in ClausChristian W. Szejnmann, Perpetrators of the Holocaust: A Historiography. In: Olaf Jensen/Claus-Christian W. Szejnmann (Hg.), Ordinary People as Mass Murderers: Perpetrators in Comparative Perspective, Basingstoke 2008, S. 25-54; Andrej Angrick, Besatzungspolitik und Massenmord. Die Einsatzgruppe D in der südlichen Sowjetunion 1941-1943, Hamburg 2003, besonders S. 386- 450.

${ }^{49}$ Michael Wildt, Volksgemeinschaft als Selbstermächtigung. Gewalt gegen Juden in der deutschen Provinz 1919 bis 1939, Hamburg 2007.

${ }^{50}$ Martina Steber, Ethnische Gewissheiten. Die Ordnung des Regionalen im bayerischen Schwaben vom Kaiserreich bis zum NS-Regime, Göttingen 2010, S. 17.

${ }^{51}$ Jensen/Szejnmann, Ordinary People as Mass Murderers.

${ }^{52}$ Vgl. H-Soz-Kult, CfP, 20.10.2011, Talking Past Each Other/Talking to Each Other: Disjunctures in Communication between German, American and Other Historians. Sektion zur Geschichte Deutschlands im 20./21. Jh. GSA Meeting 2012 - Milwaukee 
(Wisconsin/U.S.A.) 10/2012. URL: http://hsozkult. geschichte.hu-

berlin.de/termine/id=17661. Abgerufen am 13.4.2012.

${ }^{53}$ Ebd.

${ }^{54}$ Peter Fritzsche, Life and death in the Third Reich, Cambridge, Mass. 2008; Saul

Friedländer, Das Dritte Reich und die Juden. Bd. 1: Die Jahre der Verfolgung 1933-1939,

München 2000; ders., Das Dritte Reich und die Juden. Bd. 2: Die Jahre der Vernichtung, 1939-1945, München 2006.

55 Detlef Schmiechen-Ackermann, Das Potenzial der Komparatistik für die NSRegionalforschung - Vorüberlegungen zu einer Typologie von NS-Gauen und ihren Gauleitern anhand der Fallbeispiele Süd-Hannover-Braunschweig, Osthannover und WeserEms. In: Jürgen John/Horst Möller/Thomas Schaarschmidt (Hg.), Die NS-Gaue. Regionale Mittelinstanzen im zentralistischen “Führerstaat”, München 2007, S. 234-253; Szejnmann, Theoretisch-methodische Chancen.

${ }^{56}$ Für dies und Folgendes vgl. Seidel, Ruhrbergbau im Zweiten Weltkrieg, S. 6-8, 15.

57 Niedersächsische Forschungskolleg, Nationalsozialistische „Volksgemeinschaft"? Konstruktion, gesellschaftliche Wirkungsmacht und Erinnerung vor Ort. In: Newsletter Nr. 1, Mai 2010, S. 5. URL: http://www.foko-ns.de/fileadmin/fokons/pdf/Newsletter_des_Niedersaechsischen_Forschungskollegs_1_.pdf. Abgerufen am 13.4.2012. Vgl. nun Detlef Schmiechen-Ackermann (Hg.), „Volksgemeinschaft”: Mythos, wirkungsmächtige soziale Verheißung oder soziale Realität im „Dritten Reich”?, Paderborn 2012, und Martina Steber/Bernhard Gotto (Hg.), A Nazi "Volksgemeinschaft"? German Society in the Third Reich, Oxford erscheint in Kürze.

${ }^{58}$ Vgl. auch Ian Kershaw, „Volksgemeinschaft". Potential und Grenzen eines neuen Forschungskonzepts. In: Vierteljahreshefte für Zeitgeschichte, 59 (2011) 1, S. 1-17, hier 4ff. ${ }^{59}$ Ebd., S. 7-9; Michael Wildt, „Volksgemeinschaft“. Eine Antwort auf Ian Kershaw. In: Zeithistorische Forschungen/Studies in Contemporary History, Online-Ausgabe, 8 (2011), H. 1. URL: http://www.zeithistorische-forschungen.de/16126041-Wildt-1-2011. Abgerufen am 13.4.2012. 
${ }^{60}$ Wildt, „Volksgemeinschaft“, S. 5. In dieser Beziehung erwarten wir mit Spannung die Ergebnisse des Niedersächsischen Forschungskollegs "Nationalsozialistische 'Volksgemeinschaft'“? Konstruktion, gesellschaftliche Wirkungsmacht und Erinnerung vor Ort', wo „im Rahmen von exemplarisch und vergleichend angelegten regionalen Fallstudien” untersucht wird, wie genau vor Ort „Volksgemeinschaft" betrieben wurde und welche inhaltlichen Bezüge dabei eine besondere Rolle spielten. URL: http://www.fokons.de/2338.html. Abgerufen am 13.4.2012.

${ }^{61}$ Markus Fleischhauer, Der NS-Gau Thüringen 1939-1945. Eine Struktur- und Funktionsgeschichte, Köln 2010. Zitat: S. 359.

$62 \mathrm{Ebd}$

${ }^{63}$ Tagungsbericht Formen informeller Kooperation in der Herrschaftspraxis des nationalsozialistischen Deutschland, 1.3.2011-3.3.2011, Potsdam. In: H-Soz-u-Kult, 15.04.2011. URL: http://hsozkult.geschichte.hu-berlin.de/tagungsberichte/id=3621. Abgerufen am 13.4.2012.

${ }^{64}$ Ebd.

${ }^{65}$ Ebd.; Niedersächsische Forschungskolleg, Nationalsozialistische „Volksgemeinschaft“? Konstruktion, gesellschaftliche Wirkungsmacht und Erinnerung vor Ort. In: Newsletter Nr. 2, Juli 2011, S. 8. ULR: http://www.foko-ns. de/fileadmin/fokons/pdf/Newsletter_des_Niedersaechsischen_Forschungskollegs_II.pdf. Abgerufen am 13.4.2012.

${ }^{66} \mathrm{~J}$ örn Brinkhus, Luftschutz und Versorgungspolitik. Regionen und Gemeinden im NS-Staat, 1942-1944/45, Gütersloh 2011, S. 322.

${ }^{67}$ Ebd.

${ }^{68}$ Manfred Gailus, Protestantismus und Nationalsozialismus. Studien zur nationalsozialistischen Durchdringung des protestantischen Sozialmilieus in Berlin, Köln 2001; Detlef Schmiechen-Ackermann, Nationalsozialismus und Arbeitermilieus. Der nationalsozialistische Angriff auf die proletarischen Wohnquartiere und die Reaktion in den 
sozialistischen Vereinen, Bonn 1998; Norbert Fasse, Katholiken und NS-Herrschaft im Münsterland. Das Amt Velen-Ramsdorf 1918-1945, Bielefeld 1996.

${ }^{69}$ Martina Steber, Ethnische Gewissheiten. Die Ordnung des Regionalen im bayerischen Schwaben vom Kaiserreich bis zum NS-Regime, Göttingen 2010. Für Folgendes siehe speziell S. 190, 316, 478-480; Zitat: S. 479.

${ }^{70}$ Caitlin E. Murdock, Tourist Landscapes and Regional Identities in Saxony, 1878-1938. In: Central European History, 40 (2007) 4, S. 589-621; Martin Ulmer, Antisemitismus in Stuttgart 1871-1933: Studien zum öffentlichen Diskurs und Alltag, Stuttgart 2011.

${ }^{71}$ Xosé-Manoel Núñez/Maiken Umbach, Hijacked Heimats: national appropriations of local and regional identities in Germany and Spain, 1930-1945. In: European Review of HistoryRevue europe' enne d'histoire, 15 (2008) 3, S. 295-316; Manfred Kittel, Provinz zwischen Reich und Republik. Politische Mentalitäten in Deutschland und Frankreich 1918-1933/36, München 2000; Eric Storm, Regionalism in History, 1890-1945: The Cultural Approach. In: European History Quarterly, 33 (2003) 2, S. 251-265; David Laven and Timothy Baycroft, Border Regions and Identity. In: European Review of History-Revue europe' enne d'histoire, $15(2008) 3$, S. 255-275.

${ }^{72}$ Für diesen Abschnitt siehe Szejnmann, Die Bedeutung der Regionalgeschichte, S. 97-100; Szejnmann, Perpetrators of the Holocaust, S. 40-43. Die beste Überblickdarstellung des “Dritten Reiches” und die besetzten Gebiete während des Zweiten Weltkrieges ist: Das Deutsche Reich und der Zweite Weltkrieg, 10 Bd., München, 1979-2008. Für Konzentrationslager siehe Wolfgang Benz/Barbara Distel (Hg.), Der Ort des Terrors. Geschichte der nationalsozialistischen Konzentrationslager, 9 Bd., München, 2005-2009. Über NS- “Euthanasie" siehe Ernst Klee, "Euthanasie" im NS-Staat: Die "Vernichtung lebensunwerten Lebens", Frankfurt a. M. 1985; Heinz Faulstich, Hungersterben in der Psychiatrie, Freiburg 1993. Über die besetzten Gebiete siehe Andrej Angrick, Besatzungspolitik und Massenmord. Die Einsatzgruppe D in der südlichen Sowjetunion 1941-1943, Hamburg 2003; Bogdan Musial, Deutsche Zivilverwaltung und Judenverfolgung im Generalgouvernement. Eine Fallstudie zum Distrikt Lublin 1939-1944, Wiesbaden, 1999; 
Bernhard Chiari, Alltag hinter der Front. Besatzung, Kollaboration und Widerstand in Weißrußland 1941-1944, Düsseldorf, 1998. Über Kollaboration siehe Leonid Rein, The Kings and the Pawns: Collaboration in Byelorussia during World War II, Oxford, 2011; Ruth B. Birn, Die Sicherheitspolizei in Estland 1941-1944. Eine Studie zur Kollaboration im Osten, Paderborn 2006; Jan T. Gross, Neighbors: The Destruction of the Jewish Community in Jedwabne, Poland, Princeton 2001; Martin Dean, Collaboration in the Holocaust: Crimes of the Local Police in Belorussia and Ukraine, 1941-44, Basingstoke 1999. Über Frauen und Geschlechterforschung siehe Regina Mühlhäuser, Eroberungen. Sexuelle Gewalttaten und intime Beziehungen deutscher Soldaten in der Sowjetunion 1941-1945, Hamburg 2010; Franka Maubach, Die Stellung halten. Kriegserfahrungen und Lebensgeschichten von Wehrmachthelferinnen, Göttingen 2009; Elizabeth Harvey, Women and the Nazi East. Agents and Witnesses of Germanization, London 2003.

${ }^{73}$ Richtungsweisend allerdings Chiari, Alltag hinter der Front.

74 Timothy Snyder, Bloodlands. Europe between Hitler and Stalin, London 2010; Dietrich Beyrau, Schlachtfeld der Diktatoren: Osteuropa im Schatten von Hitler und Stalin, Göttingen 2000.

${ }^{75}$ Schreiber, Elite im Verborgenen, S. 13. Beispielsweisend Michael Wildt, Generation des Unbedingten. Das Führungskorps des Reichssicherheitshauptamtes, Hamburg 2002.

${ }^{76}$ Schreiber, Elite im Verborgenen, S. 15.

77 Ebd., S. 17.

${ }^{78}$ Ebd., S. 448.

${ }^{79}$ Für Folgendes siehe ebd., S. 447f, 454.

${ }^{80}$ Ebd., S. 454.

${ }^{81}$ Für das Argument der „Konsensdiktatur“ siehe Andreas Ruppert/Hansjörg Riechert (Hg.) , Herrschaft und Akzeptanz. Der Nationalsozialismus in Lippe während der Kriegsjahre. Analyse und Dokumentation, Opladen 1998. Für eine Kritik dieser Interpretation siehe Richard Evans, Coercion and Consent in Nazi Germany. In: Proceedings of the British Academy, 151 (2006), S. 53-81; Jill Stephenson, Hitler's Home Front. Württemberg under 
the Nazis, New York 2006; Götz Aly (Hg.), Volkes Stimme. Skepsis und Führervertrauen im Nationalsozialismus, Bonn 2006.

${ }^{82}$ Für die Zentralität von Rasse im Dritten Reich siehe Fritzsche, Life and Death in the Third Reich. Für die Betonung von materialistischen Aspekten für das Verhalten der Bevölkerung siehe Stöver, Volksgemeinschaft im Dritten Reich, S. 342.

${ }^{83}$ Friedländer, Die Jahre der Vernichtung; Frank Bajohr/Dieter Pohl, Massenmord und schlechtes Gewissen. Die deutsche Bevölkerung, die NS-Führung und der Holocaust, München 2006; Peter Longerich, „Davon haben wir nichts gewusst!“ Die Deutschen und die Judenverfolgung 1933-1945, München 2006.

${ }^{84}$ Bernhard Dörner, Die Deutschen und der Holocaust. Was niemand wissen wollte, aber jeder wissen konnte, Berlin 2007; Eric Johnson/Karl-Heinz Reuband, What we knew. Terror, Mass Murder and Everyday Life in Nazi Germany. An Oral History, New York 2005; David Bankier, Die öffentliche Meinung im Hitler-Staat. Die „Endlösung“ und die Deutschen. Eine Berichtigung, Berlin 1995.

${ }^{85}$ Wehler, Gesellschaftsgeschichte, S. 771.

${ }^{86}$ Forum. Everyday life in Nazi Germany. In: German History 27 (2009) 4, S. 560-579, hier 562 (vgl. Beitrag von Paul Steege; Übersetzung CCWS). 